ARTICLE

Received 27 Jan 2015 | Accepted 10 Jun 2015 | Published 16 Jul $2015 \quad$ DOl: 10.1038/ncomms8786 OPEN

\title{
Rational design of crystalline supermicroporous covalent organic frameworks with triangular topologies
}

Sasanka Dalapati ${ }^{1}$, Matthew Addicoat ${ }^{2}$, Shangbin Jin ${ }^{1}$, Tsuneaki Sakurai ${ }^{3}$, Jia Gao ${ }^{1}$, Hong Xu', Stephan Irle ${ }^{2}$, Shu Seki ${ }^{3} \&$ Donglin Jiang ${ }^{1}$

Covalent organic frameworks (COFs) are an emerging class of highly ordered porous polymers with many potential applications. They are currently designed and synthesized through hexagonal and tetragonal topologies, limiting the access to and exploration of new structures and properties. Here, we report that a triangular topology can be developed for the rational design and synthesis of a new class of COFs. The triangular topology features small pore sizes down to $12 \AA$, which is among the smallest pores for COFs reported to date, and high $\pi$-column densities of up to $0.25 \mathrm{~nm}^{-2}$, which exceeds those of supramolecular columnar $\pi$-arrays and other COF materials. These crystalline COFs facilitate $\pi$-cloud delocalization and are highly conductive, with a hole mobility that is among the highest reported for COFs and polygraphitic ensembles.

\footnotetext{
${ }^{1}$ Department of Materials Molecular Science, Institute for Molecular Science, National Institutes of Natural Sciences, 5-1 Higashiyama, Myodaiji, Okazaki 444-8787, Japan. ${ }^{2}$ Department of Chemistry, WPI-Research Initiative Institute of Transformative Bio-Molecules, Graduate School of Science, Nagoya University, Furo-cho, Chikusa-ku, Nagoya 464-8602, Japan. ${ }^{3}$ Department of Molecular Engineering, Graduate School of Engineering, Kyoto University, A4 Kyoto University Katsura Campus, Nishikyo-ku, Kyoto 615-8510, Japan. Correspondence and requests for materials should be addressed to D.J. (email: jiang@ims.ac.jp).
} 
C ovalent organic frameworks (COFs) are crystalline porous polymers with periodic ordering of organic building blocks ${ }^{1-4}$ and exhibit great potential for applications in gas storage $e^{5,6}$, manufacture of semiconductive and photoconductive devices ${ }^{7-12}$, energy conversion ${ }^{10,12,13}$ and storage $\mathrm{e}^{14,15}$, and heterogeneous catalysis ${ }^{16-19}$. The key to the design of COFs is their topology diagram, which defines the polygon size and shape and determines the crystal lattice. Hexagonal and tetragonal topologies have been exploited for the design of two-dimensional (2D) COFs with discrete pores and ordered skeletons ${ }^{1-34}$. The introduction of new topologies is highly desired to broaden the structural diversity and application of COF materials.

The triangular topology consists of $C_{6}$-geometric vertices, which is the highest symmetry of a benzene system and has a potential to efficiently exploit space for titling organic units into a crystal lattice. With this geometry, a triangular COF would feature the smallest pore size and most dense $\pi$-columns among all of the 2D COF materials. Heine et al. theoretically predicted the presence of a triangular COF structure in 2010 (ref. 30). However, the triangular topology has not been reported for the practical synthesis of COFs.

Here, we report on the rational design and synthesis of a new class of COFs based on the triangular topology. We demonstrate the synthesis of two triangular COFs (defined as HPB-COF and HBC-COF) by using two different $C_{6}$-symmetric vertices-one is hexaphenylbenzene (HPB), which is a typical propeller-shaped $\pi$-unit, and the other is hexabenzocoronene (HBC), which is a large graphitic $\pi$-unit-for Schiff-base polymerization with $\mathrm{C}_{2}$-symmetric benzene linkers. These triangular COFs form supermicropores with pore sizes of as low as $12 \AA$, which is among the lowest reported for COFs, whereas the density of $\pi$-columns can reach $0.25 \mathrm{~nm}^{-2}$, which exceeds those of the COFs and supramolecular $\pi$-arrays reported to date. These crystalline COFs exhibit excellent thermal and solvent stabilities. We demonstrate that the triangular COFs enable both intra- and inter-layer $\pi$-cloud delocalization and exhibit a prominent photoconductivity, with carrier mobilities as high as $0.7 \mathrm{~cm}^{2} \mathrm{~V}^{-1} \mathrm{~s}^{-1}$, which is among the highest reported for $2 \mathrm{D}$ COFs and polygraphitic ensembles. These results suggest that the triangular topology is useful for designing COFs with unique structures to be used in a wide variety of applications, such as gas storage, catalysis and the manufacture of sensing and semiconducting devices.

\section{Results}

Design and features of the triangular topology. Figure 1 shows the topology diagrams used for designing the 2D COFs. The triangular topology features the smallest pore size and highest $\pi$-column density among all of the topologies. The hexagonal topology leads to a pore size of $\sqrt{ } 3 L(\sim 1.732 L)$, whereas the tetragonal topology yields a pore size of $L$, as the length between two vertices is defined as $L$ (Fig. 1a). By contrast, the triangular topology generates a smaller pore diameter of only $L / \sqrt{ } 3$ $(\sim 0.58 \mathrm{~L})$, which is approximately half and one-third of those of the tetragonal and hexagonal COFs, respectively. This is the reason why the $2 \mathrm{D}$ COFs synthesized to date using the hexagonal and tetragonal topologies typically possess large pores with sizes in the mesoporous range $(>2 \mathrm{~nm})$. The introduction of a topology that directly forms microporous COFs (pore size $<2$ $\mathrm{nm}$ ) is highly desired. The microporous COFs are useful for gas storage ${ }^{35}$. We demonstrate that the triangular topology offers a straightforward strategy for the synthesis of microporous COFs. Another character of the triangular topology is that it provides the highest density of the $\pi$-columns among $2 \mathrm{D}$ COFs (Fig. 1a). The density of the $\pi$-columns is $2 / \sqrt{ } 3 L^{-2}\left(\sim 1.15 L^{-2}\right)$,
$L^{-2}$ and $4 \sqrt{3} 3 / 9 L^{-2}\left(\sim 0.77 L^{-2}\right)$, for the triangular, tetragonal and hexagonal topologies, respectively. Semiconducting and optoelectronic devices prefer ordered and dense $\pi$-units to achieve high performance.

Two $\mathrm{C}_{6}$-symmetric molecules, that is, $\left[\mathrm{H}_{2} \mathrm{~N}\right]_{6} \mathrm{HPB}$ and $\left[\mathrm{H}_{2} \mathrm{~N}\right]_{6} \mathrm{HBC}$, bearing six amino groups were synthesized as the building blocks for the vertices of triangular COFs. $\left[\mathrm{H}_{2} \mathrm{~N}\right]_{6} \mathrm{HPB}$ is a typical propeller-shaped molecule with a twisted angle of $62^{\circ}$ between the six phenyl units and focal benzene ring (Fig. 1c). $\left[\mathrm{H}_{2} \mathrm{~N}\right]_{6} \mathrm{HBC}$ is a graphitic molecule with a less twisted angle of $45^{\circ}$ between the six phenyl groups and focal $\mathrm{HBC}$ moiety (Fig. 1e). $\left[\mathrm{H}_{2} \mathrm{~N}\right]_{6} \mathrm{HPB},\left[\mathrm{H}_{2} \mathrm{~N}\right]_{6} \mathrm{HBC}$ and their intermediates were unambiguously characterized by NMR spectroscopy and mass spectrometry (Supplementary Figs 1-9). The amino groups of $\left[\mathrm{H}_{2} \mathrm{~N}\right]_{6} \mathrm{HPB}$ and $\left[\mathrm{H}_{2} \mathrm{~N}\right]_{6} \mathrm{HBC}$ reacted with benzaldehyde to form imine linkages (Fig. 1b,d, Supplementary Methods). By replacing the monofunctional benzaldehyde with a bifunctional aldehyde, that is, $C_{2}$-symmetric terephthalaldehyde, polymerization with $\left[\mathrm{H}_{2} \mathrm{~N}\right]_{6} \mathrm{HPB}$ or $\left[\mathrm{H}_{2} \mathrm{~N}\right]_{6} \mathrm{HBC}$ yielded a triangular $\mathrm{COF}$, in which the HPB or HBC unit occupies the vertices of imine-linked triangular polygons (Fig. 1b,d). HPB-COF and HBC-COF are supermicroporous polymers with pore sizes of 1.2 and $1.8 \mathrm{~nm}$, respectively (Fig. 1b,d). The triangular topology yields a $\pi$-column density of as high as $0.25 \mathrm{~nm}^{-2}$ (Table 1 ), which is the highest among 2D COFs and also exceeds that of supramolecular columnar $\pi$-arrays ${ }^{35-37}$.

Synthesis and characterization. HPB-COF and HBC-COF (Fig. 1b,d) were synthesized via the polymerization of terephthalaldehyde with $\left[\mathrm{H}_{2} \mathrm{~N}\right]_{6} \mathrm{HPB}$ and $\left[\mathrm{H}_{2} \mathrm{~N}\right]_{6} \mathrm{HBC}$ under solvothermal conditions. We screened reaction conditions, including solvent, catalyst concentration, reaction temperature and reaction time (Supplementary Figs 10-12, Supplementary Tables 1 and 2). For the optimal conditions, HPB-COF was synthesized in toluene/3 M AcOH $(20 / 1 \mathrm{v} / \mathrm{v})$ at $120^{\circ} \mathrm{C}$ for 9 days and obtained as yellow solid in a $70 \%$ isolated yield. $\mathrm{HBC}-\mathrm{COF}$ was synthesized in dioxane/ $n$-butanol/6 M AcOH $(19 / 1 / 1 \mathrm{v} / \mathrm{v})$ at $120^{\circ} \mathrm{C}$ for 12 days and obtained as deep red powder in a $95 \%$ isolated yield. $\mathrm{HPB}-\mathrm{COF}$ and $\mathrm{HBC}-\mathrm{COF}$ were unambiguously characterized by various analytical methods (Supplementary Figs 10-21).

Infrared spectroscopy exhibited a typical stretching vibration band at $1,622-1,626 \mathrm{~cm}^{-1}$, which was assigned to the $\mathrm{C}=\mathrm{N}$ bond and also confirmed by the model compounds of HPB-Ph and HBC-Ph (Supplementary Figs 13 and 14). HPB-COF exhibited a micrometre-scale sheet-shape morphology, as revealed by fieldemission scanning electron microscopy, whereas HBC-COF adopted a particle morphology (Supplementary Fig. 15). Highresolution transmission electron microscopy revealed the presence of layered and triangular textures (Supplementary Fig. 16).

Crystal structure. Crystal structures of HPB-COF and HBC-COF were resolved by using $\mathrm{X}$-ray diffraction (XRD) measurements in conjunction with Pawley refinements and structural simulations (Figs 2 and 3 and Supplementary Methods) ${ }^{38-40}$. HPB-COF exhibited XRD signals at $4.85^{\circ}$ and $9.70^{\circ}$, which were assigned to the (100) and (200) facets, respectively (Fig. 2a, red curve). The presence of the (001) facet at $19.45^{\circ}$ indicates that the periodicity of the $2 \mathrm{D}$ sheets is extended to the third dimension. The Pawley refinements (green curve) reproduced the experimentally observed XRD pattern with a negligible difference (black curve), confirming the correctness of the above peak assignments. HBCCOF exhibited XRD peaks at $3.4^{\circ}, 6.8^{\circ}$ and $26.26^{\circ}$ that were assigned to the (100), (200) and (001) facets, respectively (Fig. 3a, red curve). The Pawley refinements yielded an XRD pattern (green curve) that is consistent with the observed XRD profile. 
a
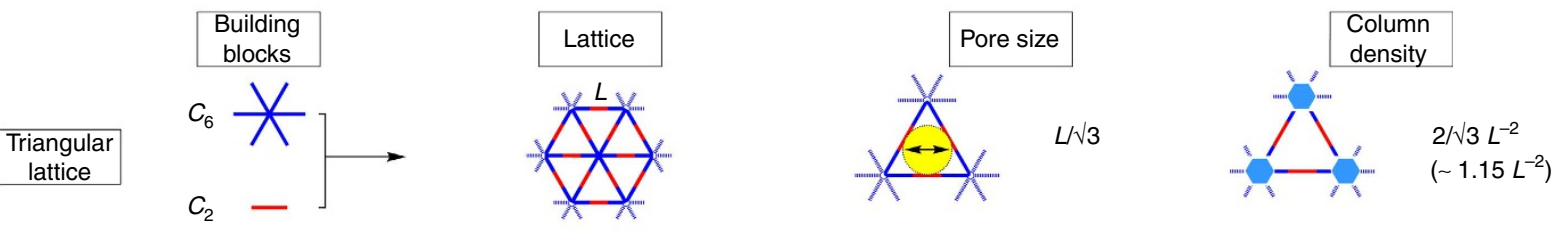

Tetragonal
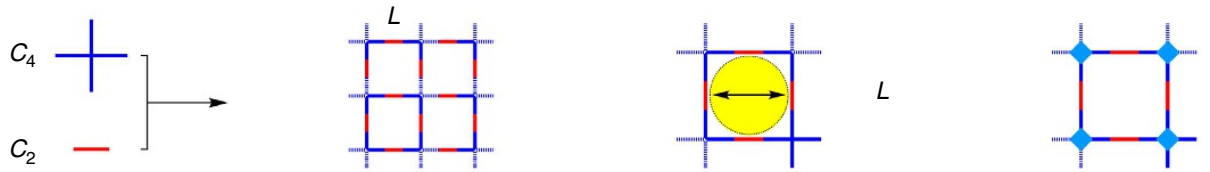

$L^{-2}$

Hexagonal lattice
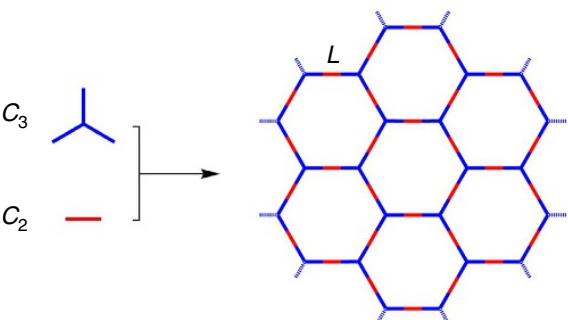

b
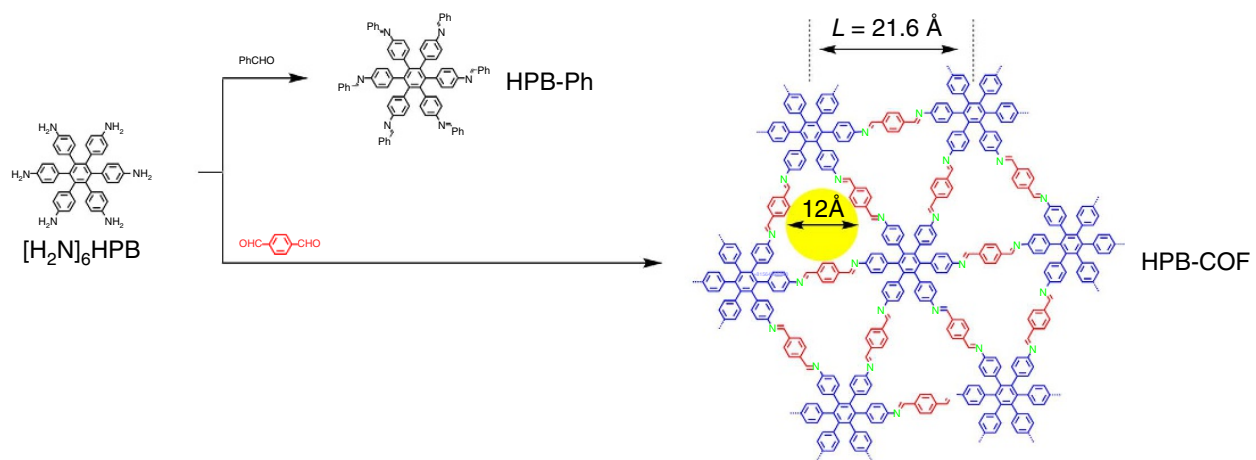

C
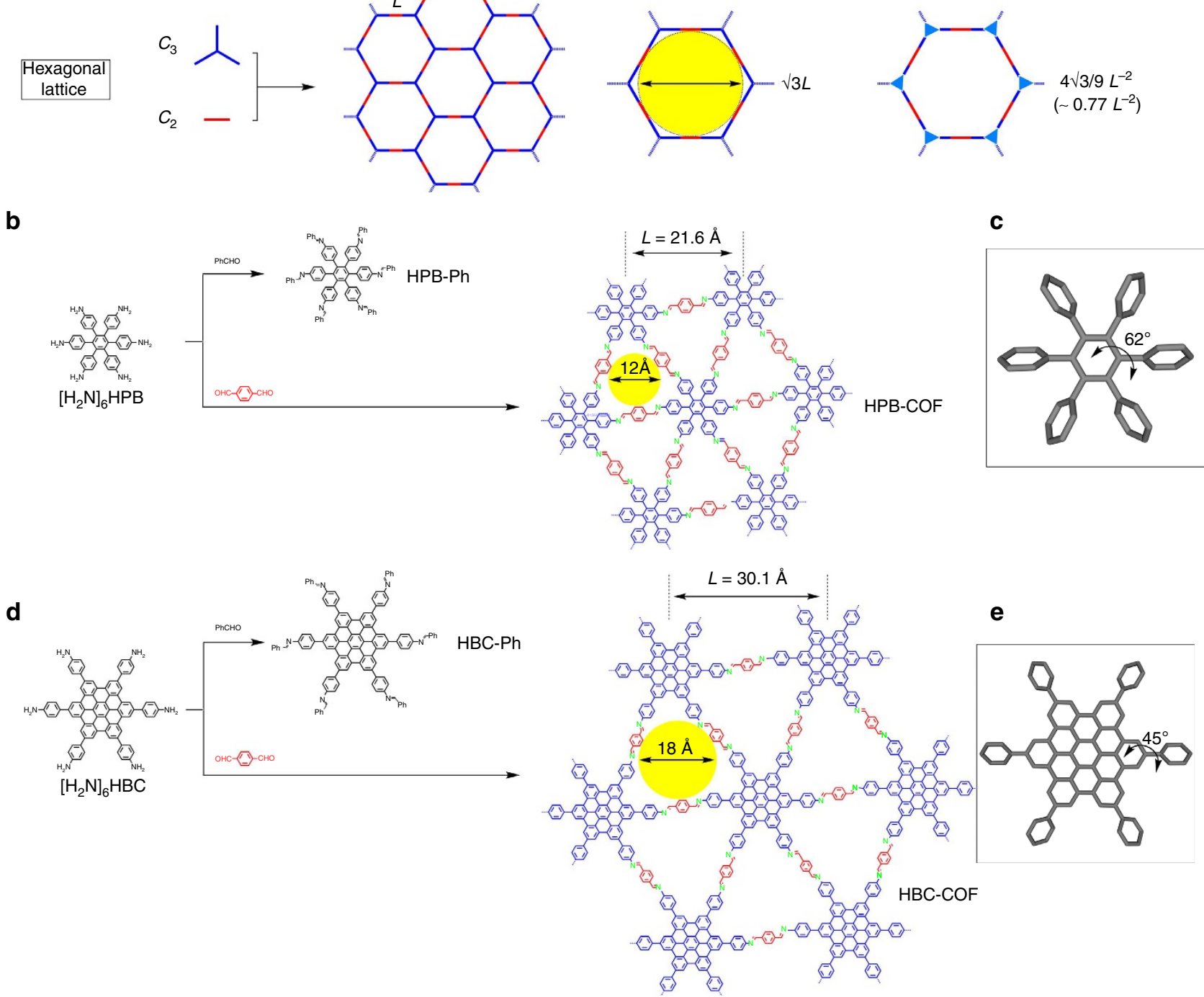

Figure 1 | Design of topology diagrams and synthesis of trigonal COFs. (a) Topology diagrams for COFs and their pore size and $\pi$-column density. (b) Schematic representation of the synthesis of imine-linked triangular HPB-COF, together with its model reaction. (c) Propeller-shaped HPB building block. (d) Schematic representation of the synthesis of imine-linked triangular HBC-COF, together with its model reaction. (e) HBC building block.

The negligible difference (black curve) assures the suitable assignment of the observed XRD signals.

To generate the optimal crystal structures, the densityfunctional tight-binding (DFTB) method including LennardJones dispersion was applied to both COFs (for details, see Supplementary Methods; http://www.dftb.org $)^{40}$. For the monolayer of HPB-COF, the optimal cell length was $a=b=21.57 \AA$, while the six phenyl units of HPB are twisted by $62^{\circ}$ with respect to the central benzene ring in a propeller-like arrangement. By contrast, the monolayer of HBC-COF exhibited an optimal cell length of $a=b=30.14 \AA$, while the six phenyl units are twisted to form a $45^{\circ}$ angle with respect to the $\mathrm{HBC}$ plane. Using these monolayer structures, three stacked configurations, that is, eclipsed AA (hybrid), slipped AA and 


\begin{tabular}{|c|c|c|c|c|c|c|}
\hline COFs & $a=b(\AA)$ & $c(\AA)$ & $\begin{array}{c}\text { Total } \\
\text { crystal } \\
\text { stacking } \\
\text { energy } \\
\left(\text { (kcal mol }^{-1}\right) \\
\end{array}$ & $\begin{array}{l}\text { Pore } \\
\text { size } \\
(n m)\end{array}$ & $\begin{array}{c}\pi \text {-Column } \\
\text { density } \\
\left(n^{-2}\right)\end{array}$ & $\begin{array}{l}\text { Band } \\
\text { gap } \\
(\mathrm{eV})\end{array}$ \\
\hline HPB-COF & 21.57 & 5.17 & 46.74 & 1.2 & 0.25 & 2.17 \\
\hline $\mathrm{HBC}-\mathrm{COF}$ & 30.14 & 3.54 & 136.37 & 1.8 & 0.13 & 1.71 \\
\hline
\end{tabular}
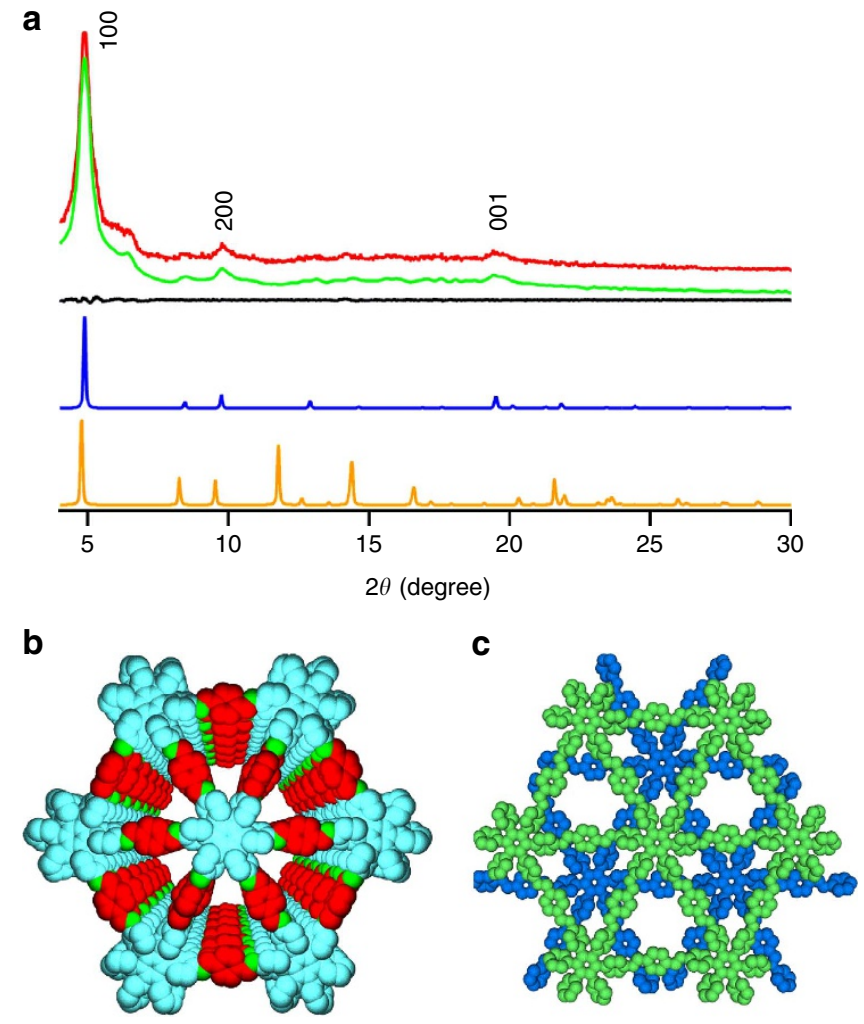

Figure 2 | Crystal structure of HPB-COF. (a) XRD patterns of experimentally observed (red curve), Pawley refined pattern (green curve), their difference (black curve), eclipsed hybrid AA stacking mode (blue curve) and staggered $A B$ stacking mode (orange curve). The crystal facets are shown with indices on the peaks. (b) View of the eclipsed hybrid AA stacking structure. (c) View of the staggered $A B$ stacking structure.

staggered $\mathrm{AB}$ modes, were generated and optimized. The interlayer stacking distances for the central ring, and the corresponding Lennard-Jones dispersion, and crystal stacking energies per monolayer of each structure are shown in the Supplementary Tables 3 and 4. For HPB-COF, the eclipsed hybrid AA stacking mode, which is situated as a tilted and planar arrangement with respect to the linkers for alternate layers, gives rise to the most stable structure as a result of favourable $\mathrm{C}$ $\mathrm{H} \cdot \cdots \pi$ interactions between two layers. For HBC-COF, the $0.8 \AA$ slipped AA stacking mode is the most stable structure. The twisted angles of the phenyl groups in the HPB and HBC units decreased to $54^{\circ}$ and $20^{\circ}$ for the eclipsed hybrid AA mode of HPB-COF and slipped AA mode of $\mathrm{HBC}-\mathrm{COF}$, respectively. Therefore, in the frameworks, the $2 \mathrm{D}$ polygon sheets become more planar as a result of interlayer $\pi-\pi$ forces. According to
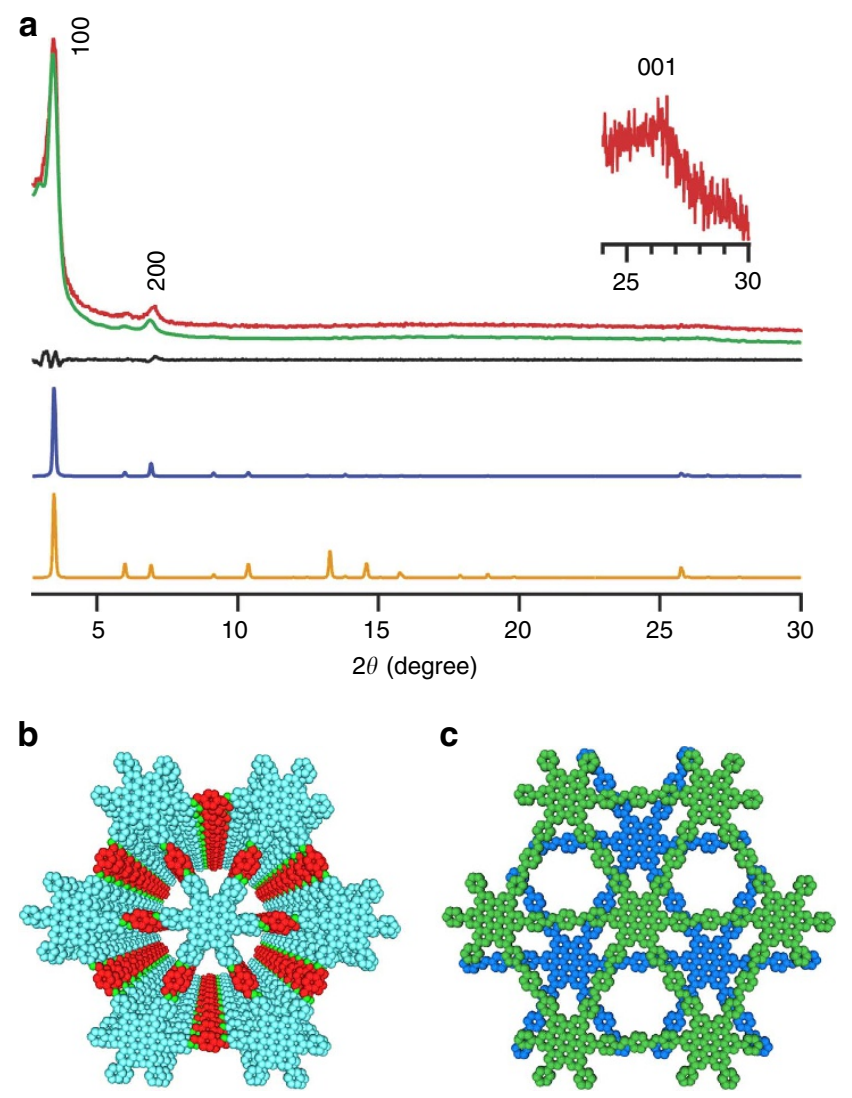

Figure 3 | Crystal structure of HBC-COF. (a) XRD patterns of

experimentally observed (red curve), Pawley refined pattern (green curve), their difference (black curve), $0.8 \AA$ slipped AA stacking mode (blue curve) and staggered $A B$ stacking mode (orange curve). The crystal facets are shown with indices on the peaks. (b) View of the $0.8 \AA$ slipped $A A$ stacking structure. (c) View of the staggered $A B$ stacking structure.

these structures, HPB-COF consists of HPB $\pi$-columns with a density of as high as $0.25 \mathrm{~nm}^{-2}$, and HBC-COF exhibits a graphitic column density of $0.13 \mathrm{~nm}^{-2}$ (Table 1). These densities are the highest among COFs and exceed that of supramolecular columnar HPB and HBC $\pi$-arrays. Such a highly dense yet ordered $\pi$-structure is intriguing for optoelectronic applications.

On the basis of the above optimal stacking structures, we deduced the crystal structures using the Reflex module package in Materials Studio ${ }^{39}$. The generated XRD patterns of the eclipsed hybrid AA stacking mode for HPB-COF (Fig. 2a, blue curve) and the $0.8 \AA$-slipped AA stacking mode for HBC-COF (Fig. 3a, blue curve) can accurately reproduce both the positions and intensities of the experimentally observed XRD peaks. Figures $2 \mathrm{~b}$ and $3 \mathrm{~b}$ illustrate the eclipsed hybrid AA and $0.8 \AA$-slipped AA stacking structures of $\mathrm{HPB}-\mathrm{COF}$ and $\mathrm{HBC}-\mathrm{COF}$, respectively. In contrast to the AA modes, the staggered $\mathrm{AB}$ modes (Figs 2a and 3a, orange curves) cannot reproduce the experimentally observed XRD patterns and half of the pores are covered by the neighbouring sheets (Figs $2 c$ and $3 c$ ).

The $C_{6}$-symmetric vertices play a vital role in controlling the interlayer interactions. For example, the HPB-COF with propeller-shaped HPB vertices has an interlayer distance of $5.17 \AA$, which is considerably larger than that (3.54 $\AA$ ) of HBC-COF. Although HPB-COF has $a$ and $b$ lengths that are smaller than HBC-COF, the total crystal stacking energy of HPB is calculated to be only $46.74 \mathrm{kcal} \mathrm{mol}^{-1}$ per monolayer (Table 1), which is only one-third that of HBC-COF $\left(136.37 \mathrm{kcal} \mathrm{mol}^{-1}\right)$. Thus, the 
propeller-shaped HPB knots offer a loose stacking, whereas the graphitic units enable a tight layering of $2 \mathrm{D}$ sheets.

Gas adsorption and porosity. To investigate the porous structure, nitrogen sorption isotherms were measured at $77 \mathrm{~K}$ (Fig. 4). HPB-COF and HBC-COF exhibited typical type I isotherms (Fig. 4a,c), which are characteristic of microporous materials. The Brunauer-Emmett-Teller (BET) surface area and pore volume for HPB-COF were calculated to be $965 \mathrm{~m}^{2} \mathrm{~g}^{-1}$ and $0.79 \mathrm{~cm}^{3} \mathrm{~g}^{-1}$, respectively. HBC-COF exhibited a BET surface area and pore volume of $469 \mathrm{~m}^{2} \mathrm{~g}^{-1}$ and $0.29 \mathrm{~cm}^{3} \mathrm{~g}^{-1}$, respectively. On the basis of the nonlocal density-functional theory calculation from the sorption curves, the pore size of HPB-CPF and HBC-COF was estimated to be 1.2 and $1.8 \mathrm{~nm}$, respectively (Fig. 4b,d). These supermicropores are among the smallest reported for $2 \mathrm{D}$ COFs.

We calculated the theoretical BET surface areas of HPB-COF and HBC-COF using the model structures of DFTB calculations and observed that HPB-COF (Supplementary Fig. 17) and HBC-COF (Supplementary Fig. 18) have theoretical surface areas of 1,588 and $1,381 \mathrm{~m}^{2} \mathrm{~g}^{-1}$, respectively. The difference between experimentally observed BET surface areas and theroretical values is related to the crystallinity of the COF samples. The high theoretical surface area of HPB-COF compared with that of $\mathrm{HBC}-\mathrm{COF}$ is cuased by a large $c$ value $(5.1 \AA$ for $\mathrm{HPB}-\mathrm{COF}$ and $3.5 \AA$ for HBC-COF) and a low monomer molecular weight (624 for the HPB monomer versus 1,068 for the HBC monomer $)^{41}$. These factors also reflect in the experimentally obsvered porosity.
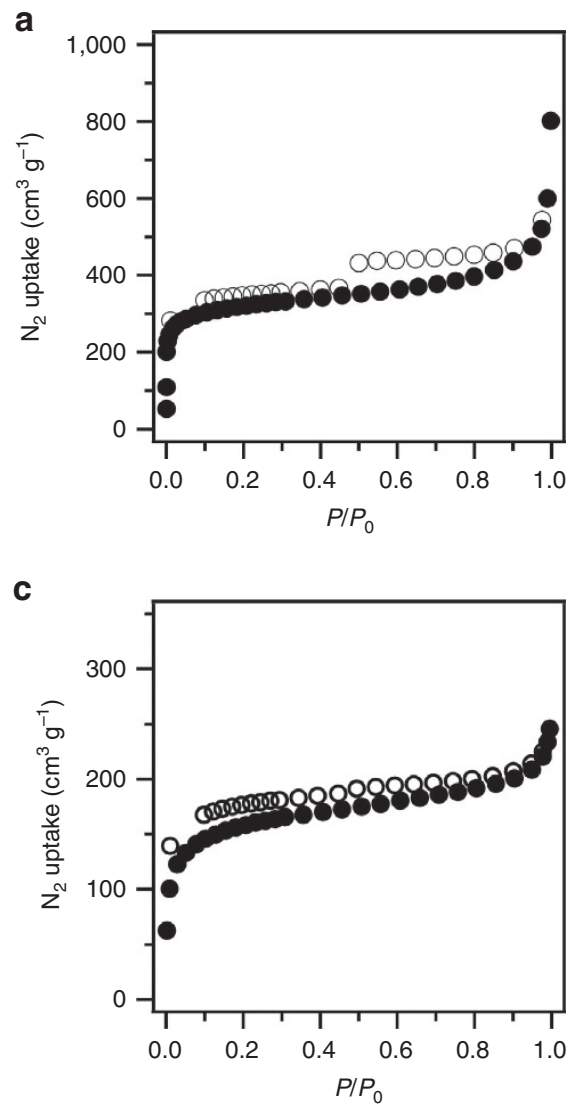

Stability. To examine the chemical stability, we dispersed the COF samples in different solvents such as hexane, tetrahydrofuran (THF), $\mathrm{MeOH}$, water and aqueous $\mathrm{HCl}(1 \mathrm{M})$ and $\mathrm{NaOH}(1 \mathrm{M})$ solutions at $25^{\circ} \mathrm{C}$ and boiling temperatures (heating at $100^{\circ} \mathrm{C}$ ) for $24 \mathrm{~h}$. Both COFs fairly retained their crystallinity (Fig. 5) and porosity (Supplementary Tables 5 and 6 and Supplementary Figs 19 and 20) upon treatment in organic solvents, such as hexane, THF and $\mathrm{MeOH}$, and water at $25^{\circ} \mathrm{C}$ and their boiling temperatures. Nevertheless, HBC-COF was unstable in aqueous $\mathrm{HCl}$ solution $(1 \mathrm{M})$ at 25 and $100^{\circ} \mathrm{C}$, as revealed by their XRD and nitrogen sorption measurements. HPB-COF was much easier to be destroyed in aqueous $\mathrm{HCl}$ solution (1 $\mathrm{M}$ ); no solid sample remained after treatment. Notably, HPB-COF and $\mathrm{HBC}-\mathrm{COF}$ were stable in aqueous $\mathrm{NaOH}(1 \mathrm{M})$ solution at $25^{\circ} \mathrm{C}$ (Fig. 5). HPB-COF kept crystallinity and porosity upon treatment in the $\mathrm{NaOH}$ solution at $100^{\circ} \mathrm{C}$, whereas $\mathrm{HBC}$-COF was unstable in the $\mathrm{NaOH}$ solution at elevated temperature. The instability is likely related to the hydrolysis of the imine linkages. We conducted thermal gravimetric analysis to investigate thermal stability and did not observed gaseous decomposition products for $\mathrm{HPB}-\mathrm{COF}$ and $\mathrm{HBC}-\mathrm{COF}$ up to 520 and $550^{\circ} \mathrm{C}$, respectively (Supplementary Fig. 21). The stability of COFs is crucial for use in various applications.

\section{Discussion}

We reported the first example of a semiconducting TP-COF in 2008 (ref. 7). In recent years, we and other groups have prepared a series of conducting COFs with ordered $\pi$-columns ${ }^{8-12,21-26,31-32}$. Compared with self-assembled systems and liquid crystals, a
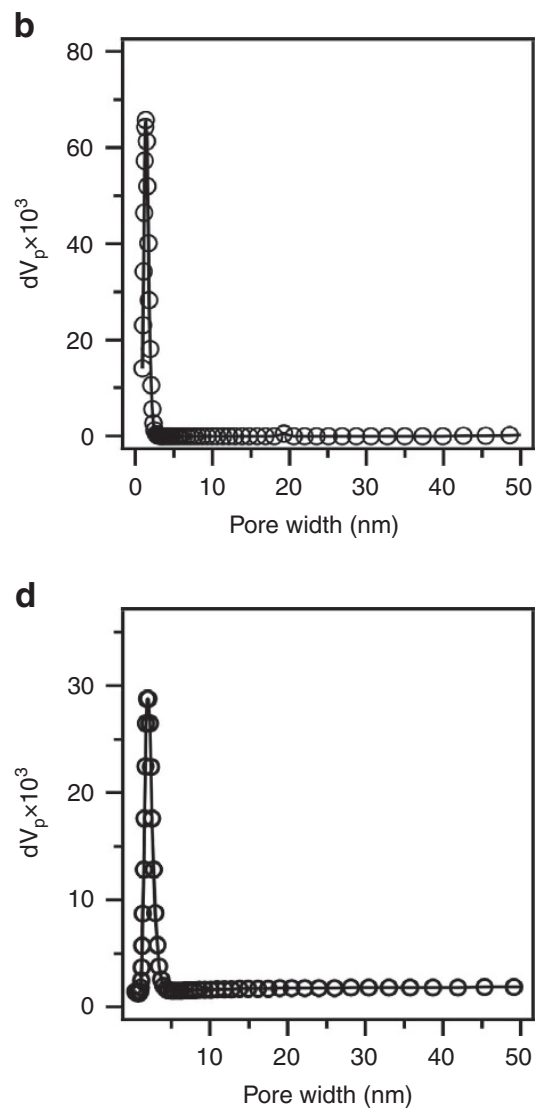

Figure 4 | Gas adsorption. (a) Nitrogen sorption isotherm curve of HPB-COF (filled circles for adsorption and open circles for desorption). (b) Pore size distribution profile of HPB-COF. (c) Nitrogen sorption isotherm curve of HBC-COF (filled circles for adsorption and open circles for desorption). (d) Pore size distribution profile of HBC-COF. 

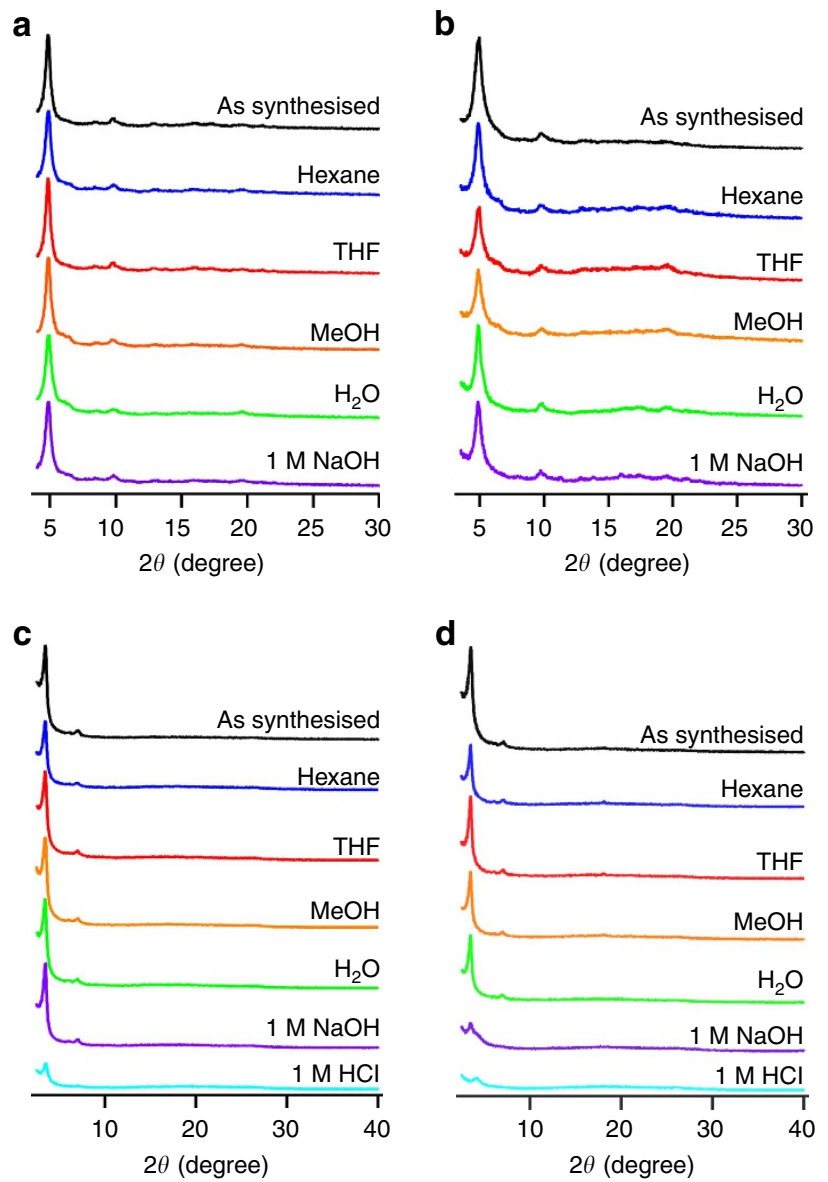

Figure 5 | Stability. (a,b) XRD patterns of HPB-COF upon 1-day treatment under different conditions at (a) $25^{\circ} \mathrm{C}$ and (b) boiling temperatures (heating at $100^{\circ} \mathrm{C}$ ). (c,d) XRD patterns of $\mathrm{HBC}$-COF upon 1-day treatment under different conditions at (c) $25^{\circ} \mathrm{C}$ and (d) boiling temperatures (heating at $100^{\circ} \mathrm{C}$ ).

distinct feature of COFs' $\pi$-columns is that they trigger significant electronic coupling allowing $\pi$-cloud delocalization, which facilitates charge transport through the $\pi$-channels. However, except for a few examples ${ }^{10,26}$, the majority of these COFs are water or moisture sensitive, restricting their device applications.

Electronic absorption spectroscopy was conducted to evaluate the $\pi$-electronic functions. Compared with the HPB monomer that exhibits an absorption band at $329 \mathrm{~nm}$, HPB-COF (yellow colour, Fig. 6a inset) exhibited a $94 \mathrm{~nm}$ redshifted absorption band centred at $423 \mathrm{~nm}$ (Fig. 6a). By contrast, HBC-COF (deep red colour, Fig. $6 \mathrm{~b}$ inset) exhibited an absorption band at $480 \mathrm{~nm}$ (Fig. 6b), which is redshifted by $55 \mathrm{~nm}$ from that of the HBC monomer $(425 \mathrm{~nm})$. Therefore, the triangular COFs constitute 2D conjugated polymer networks, which allow for extended $\pi$-cloud delocalization over the ordered frameworks.

To obtain insight into the electronic properties of HPB-COF and $\mathrm{HBC}-\mathrm{COF}$, we evaluated the highest occupied molecular orbital (HOMO) and the lowest unoccupied molecular orbital (LUMO) energy gaps using DFTB calculations (Supplementary Tables 3 and 4). For HPB-COF, the monolayer has a HOMOLUMO gap of $2.30 \mathrm{eV}$, whereas upon eclipsed hybrid AA stacking the gap decreased to $2.17 \mathrm{eV}$. The monolayer of $\mathrm{HBC}-\mathrm{COF}$ has a small gap of $2.00 \mathrm{eV}$, which is further decreased to $1.71 \mathrm{eV}$ for the $0.8 \AA$ slipped AA stacking structure (Table 1). A low energy gap facilitates charge carrier transport in the frameworks; therefore, HBC-COF is superior to HPB-COF in charge carrier transport as evidenced by its low energy gaps. The triangular topology allows for intra-sheet $\pi$-cloud delocalization, whereas the $\pi$-columns of $C_{6}$-symmetric vertices further promote inter-sheet $\pi$-cloud delocalization. Frontier orbital mapping suggests that the HOMO is centred on the HPB and HBC vertices, whereas the LUMO is located on the phenyl edges (Supplementary Fig. 22).

We further investigated the photoconductivity of HPB-COF and $\mathrm{HBC}-\mathrm{COF}$ using the flash photolysis time-resolved microwave conductivity (FP-TRMC) method ${ }^{42,43}$. Upon excitation with a 355-nm laser pulse at a photon density of $9 \times 10^{15}$ photons $\mathrm{cm}^{-}$ 2 , HPB-COF under air yielded a $\phi \Sigma \mu$ value $(\phi$ : photocarrier generation quantum yield, $\Sigma \mu$ : the sum of charge carrier mobility) of $0.8 \times 10^{-5} \mathrm{~cm}^{2} \mathrm{~V}^{-1} \mathrm{~s}^{-1}$ (Fig. 6c). By contrast, under otherwise identical conditions, HBC-COF exhibited a $\phi \Sigma \mu$ value of $1.5 \times 10^{-5} \mathrm{~cm}^{2} \mathrm{~V}^{-1} \mathrm{~s}^{-1}$ (Fig. 6d), which is twice that of HPBCOF. The $\phi \Sigma \mu$ value of HBC-COF is one to two orders of magnitude higher than those of representative conducting tetrathiafulvalene $\mathrm{COFs}^{26}$. After the initial time, a decay for both cases can be assumed due to charge re-combinations, whereas the retained tails suggest the presence of long-lived charges in the COFs.

To evaluate the hole mobility of $\mathrm{HBC}-\mathrm{COF}$, the $\varphi$ value was determined by the direct current integration method ${ }^{42-44}$. The HBC-COF on comb-type electrode devices was exposed to a $355-\mathrm{nm}$ laser pulse at $9.1 \times 10^{15}$ photons $\mathrm{cm}^{-2}$, and the time-offlight transient photocurrent was monitored at different bias voltages (Fig. 6e). A linear $I-V$ curve indicates ohmic behaviour for the electric conduction of HBC-COF (Fig. 6f). By comparing the photocurrents with those of poly $\left(9,9^{\prime} \text {-dioctylfluorene }\right)^{42}$, the $\phi$ value of $\mathrm{HBC}-\mathrm{COF}$ was estimated to be $2.1 \times 10^{-5}$. The combination of these experiments allows for the calculation of an intrinsic hole mobility as high as $0.7 \mathrm{~cm}^{2} \mathrm{~V}^{-1} \mathrm{~s}^{-1}$. Notably, this hole mobility is $40-70$-fold higher than those of triphenylenebased COF $\left(0.01 \mathrm{~cm}^{2} \mathrm{~V}^{-1} \mathrm{~s}^{-1}\right)^{32}$ and porphyrin-knoted $\mathrm{ZnP}-\mathrm{COF}$ $\left(0.016 \mathrm{~cm}^{2} \mathrm{~V}^{-1} \mathrm{~s}^{-1}\right)^{25}$, and is among the highest reported for $2 \mathrm{D}$ $\mathrm{COFs}^{10,21,22,24}$. These results indicate that the triangular yet stable COFs can be used in semiconducting devices.

In summary, we have developed a triangular topology for the design and synthesis of an unprecedented class of COFs. From a porous material point of view, the triangular topology provides a straightforward method of preparing microporous 2D COFs. From a $\pi$-electronic perspective, the triangular topology enables the periodic ordering of $\pi$-columnar arrays at the highest density. As demonstrated by HPB-COF and $\mathrm{HBC}-\mathrm{COF}$, the triangular COFs offer crystallinity, microporosity, stability, $\pi$-cloud delocalization and photoconductivity; the materials with these multifunctions may find a wide variety of applications, such as gas storage, size-selective separation, catalysis and the manufacture of sensing, semiconducting and optoelectronic devices. Thus, this study opens a new chemical approach to the structural and functional design of crystalline porous organic materials.

\section{Methods}

Synthesis of HPB-COF. A $10-\mathrm{ml}$ Pyrex tube was charged with $\left[\mathrm{NH}_{2}\right]_{6} \mathrm{HPB}$ $(10.0 \mathrm{mg}, 0.0094 \mathrm{mmol})$, toluene $(2 \mathrm{ml})$, terephthaldehyde $(6.44 \mathrm{mg}, 0.05 \mathrm{mmol})$ and $0.1 \mathrm{ml} \mathrm{AcOH}(3 \mathrm{M})$. The mixture was degassed through three freeze-pump-thaw cycles, sealed under vacuum and heated at $120^{\circ} \mathrm{C}$ for 9 days. The mixture was cooled to room temperature and the yellow precipitate was collected by centrifugation, washed with tetrahydrofuran several times, and dried under vacuum for $12 \mathrm{~h}$ to afford HPB-COF in a $70 \%$ isolated yield. Other reaction conditions (Supplementary Table 1, Supplementary Figs 10 and 12), such as toluene/6 M AcOH (20/1 v/v), toluene/dioxane/3 M AcOH (19/1/1 v/v) and toluene/dioxane/ $6 \mathrm{M} \mathrm{AcOH}(19 / 1 / 1 \mathrm{v} / \mathrm{v})$, followed the same experimental procedure.

Synthesis of HBC-COF. A 10 -ml Pyrex tube was charged with $\left[\mathrm{NH}_{2}\right]_{6} \mathrm{HBC}$ (10.0 mg, $0.0094 \mathrm{mmol})$, dioxane $/ n$-butanol $(2 \mathrm{ml}, 19 / 1 \mathrm{v} / \mathrm{v})$, terephthaldehyde $(3.76 \mathrm{mg}, 0.028 \mathrm{mmol})$ and $0.1 \mathrm{ml} \mathrm{AcOH}(6 \mathrm{M})$. The mixture was degassed through three freeze-pump-thaw cycles, sealed under vacuum and heated 
a

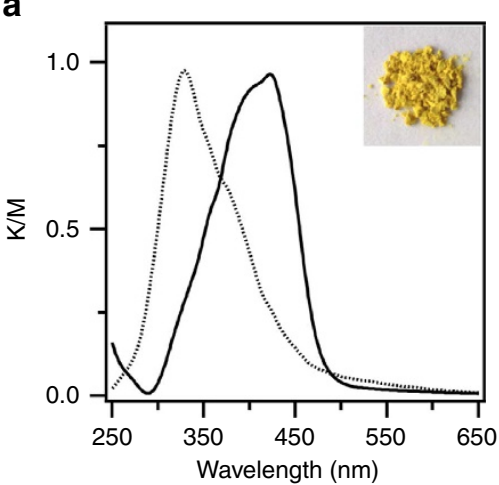

C

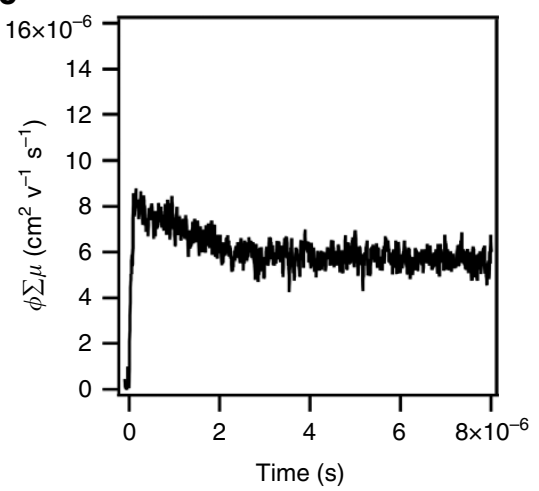

e

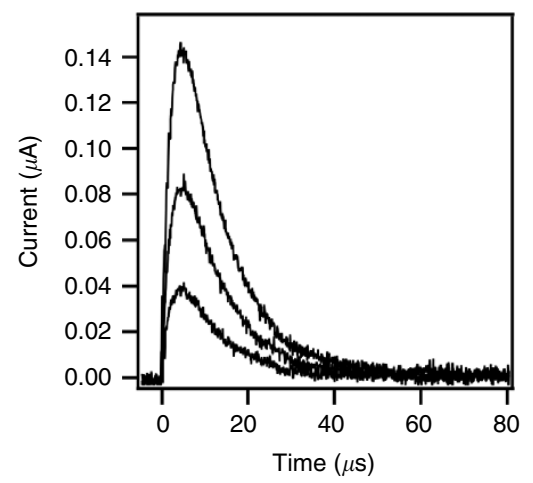

b

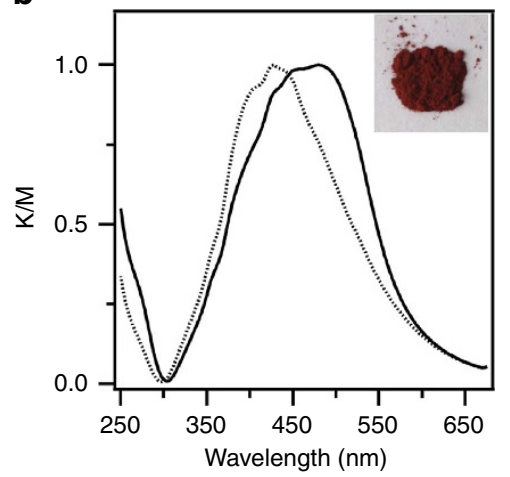

d

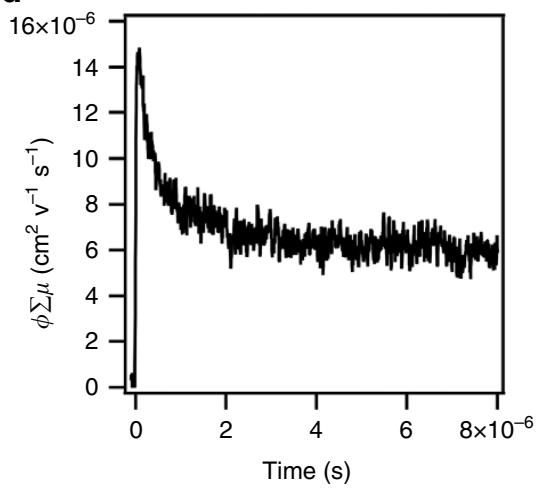

f

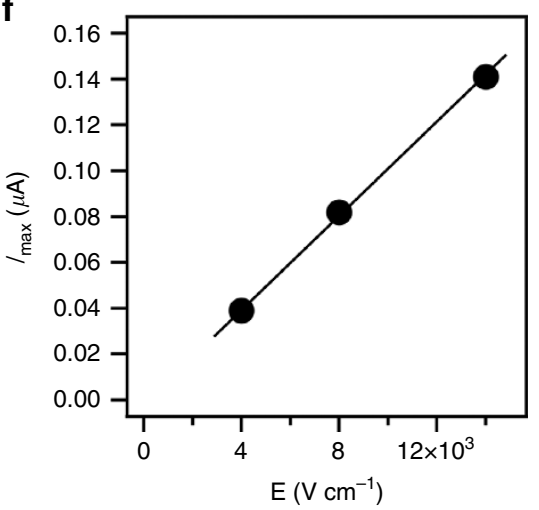

Figure 6 | $\pi$-Electronic and conducting properties. (a) Solid-state electronic absorption spectra of HPB-COF (solid curve) and HPB (dotted curve). Inset: a photo of the HPB-COF sample. (b) Solid-state electronic absorption spectra of HBC-COF (solid curve) and HBC (dotted curve). Inset: a photo of the HBCCOF sample. (c) FP-TRMC profile of HPB-COF. (d) FP-TRMC profile of HBC-COF. (e) Photocurrent generation of spin coated HBC-COF on a comb-type gold electrode device (electrode gap $=5 \mu \mathrm{m}$ ) at different bias voltages $(2,4$ and $7 \mathrm{~V}$ ). (f) I-V curve of HBC-COF on the comb-type gold electrode device.

at $120^{\circ} \mathrm{C}$ for 12 days. The reaction mixture was cooled to room temperature and the deep red precipitate was collected by centrifugation, washed with THF several times, and dried under vacuum for $12 \mathrm{~h}$ to afford $\mathrm{HBC}-\mathrm{COF}$ in a $95 \%$ isolated yield. Other solvent conditions (Supplementary Table 2, Supplementary Figs 11 and 12), such as toluene/dioxane/6 M AcOH (1/19/1 v/v), mesitylene/dioxane/6 M AcOH (1/19/1 $\mathrm{v} / \mathrm{v})$, mesitylene/THF/6 M AcOH (15/5/1 v/v) and $o$-DCB/THF/6 M AcOH (15/5/1 $\mathrm{v} / \mathrm{v}$ ), followed the same experimental procedure.

FP-TRMC. The charge carrier mobility was measured by the FP-TRMC technique at $20^{\circ} \mathrm{C}$ under air. Film samples on a quartz plate were prepared by drop-casting a toluene solution that contains HPB-COF or HBC-COF together with polystyrene. Charge carriers were photochemically generated using the third harmonic generation $(\lambda=355 \mathrm{~nm})$ of a Spectra Physics model INDI-HG Nd:YAG laser with a pulse duration of 5-8 ns. The photon density of the $355-\mathrm{nm}$ pulse was $9.0 \times 10^{15}$ photons $\mathrm{cm}^{-2}$. The microwave frequency and power were set at $\sim 9.1 \mathrm{GHz}$ and $3 \mathrm{~mW}$, respectively. The TRMC signal, picked up by a diode (rise time $<1 \mathrm{~ns}$ ), was monitored with a Tektronics model TDS3052B digital oscilloscope. The observed conductivities were normalized, based on the photocarrier generation yield $(\phi)$ multiplied by sum of the charge carrier mobilities $(\Sigma \mu)$, according to the equation, $\phi \Sigma \mu=\left(1 / e \mathrm{~A} I_{0} F_{\text {light }}\right)\left(\Delta P_{\mathrm{r}} / P_{\mathrm{r}}\right)$, where $e, \mathrm{~A}, I_{0}, F_{\text {light }}, P_{\mathrm{r}}$ and $\Delta P_{\mathrm{r}}$ are the unit charge of a single electron, sensitivity factor $\left(\mathrm{S}^{-1} \mathrm{~cm}^{-1}\right)$, incident photon density of the excitation laser (photon $\mathrm{cm}^{-2}$ ), correction (or filling) factor $\left(\mathrm{cm}^{-1}\right)$, and reflected microwave power and its change, respectively.

Current integration method. The $\phi$ values were determined by the direct current integration method at $20^{\circ} \mathrm{C}$. An interdigitated comb-type gold electrode device with $5 \mu \mathrm{m}$ gaps, $40 \mathrm{~nm}$ height and $2 \mathrm{~mm}$ width was fabricated by a lithographic process on a glass substrate. Samples were fabricated by spin-coating a toluene suspension of finely grounded $\mathrm{HBC}-\mathrm{COF}$ on the electrodes. The samples were exposed to a 355 -nm pulse with $9.0 \times 10^{15}$ photons $\mathrm{cm}^{-2}$. Current transients were monitored by a Tektronix model TDS3052B digital oscilloscope equipped with a $10 \mathrm{k} \Omega$ termination resistance. The applied voltage was set at 2,4 and $7 \mathrm{~V}$ by an Advantest model R8252 digital electrometer. Using a $\phi$ value of $2.3 \times 10^{-4}$ for poly(9,9'-dioctylfluorene) film as a reference, the $\phi$ values of $\mathrm{HBC}$-COF were estimated by comparing the photocurrent peaks with those of poly $\left(9,9^{\prime}\right.$-dioctylfluorene) under the same bias voltage. 


\section{References}

1. Côté, A. P. et al. Porous, crystalline, covalent organic frameworks. Science 310, 1166-1170 (2005).

2. Feng, X., Ding, X. \& Jiang, D. Covalent organic frameworks. Chem. Soc. Rev. 41, 6010-6022 (2012).

3. Ding, S.-Y. \& Wang, W. Covalent organic frameworks (COFs): from design to applications. Chem. Soc. Rev. 42, 548-568 (2013).

4. Wu, D. et al. Design and preparation of porous polymers. Chem. Rev. 112, 3959-4015 (2012)

5. Furukawa, H. \& Yaghi, O. M. Storage of hydrogen, methane, and carbon dioxide in highly porous covalent organic frameworks for clean energy applications. J. Am. Chem. Soc. 131, 8875-8883 (2009).

6. Tilford, R. W., Mugavero, III S. J., Pellechia, P. J. \& Lavigne, J. J. Tailoring microporosity in covalent organic frameworks. Adv. Mater. 20, 2741-2746 (2008)

7. Wan, S., Guo, J., Kim, J., Ihee, H. \& Jiang, D. A belt-shaped, blue luminescent, and semiconducting covalent organic framework. Angew. Chem. Int. Ed. 47, 8826-8830 (2008)

8. Colson, J. W. et al. Oriented 2D covalent organic framework thin films on single-layer graphene. Science 332, 228-231 (2011).

9. Dogru, M. et al. A photoconductive thienothiophene-based covalent organic framework showing charge transfer towards included fullerene. Angew. Chem. Int. Ed. 52, 2920-2924 (2013).

10. Guo, J. et al. Conjugated organic framework with three-dimensionally ordered stable structure and delocalised $\pi$ clouds. Nat. Commun. 4, 2736 (2013).

11. Bertrand, G. H. V., Michaelis, V. K., Ong, T., Griffin, R. G. \& Dincă, M. Thiophene-based covalent organic frameworks. Proc. Nat. Acad. Sci. USA 110, 4923-4928 (2013).

12. Calik, M. et al. Extraction of photogenerated electrons and holes from a covalent organic framework integrated heterojunction. J. Am. Chem. Soc. 136, 17802-17807 (2014)

13. Stegbauer, L., Schwinghammer, K. \& Lotsch, B. V. A hydrazone-based covalent organic framework for photocatalytic hydrogen production. Chem. Sci. 5, 2789-2793 (2014)

14. Xu, F. et al. Electrochemically active, crystalline, mesoporous covalent organic frameworks on carbon nanotubes for synergistic lithium-ion battery energy storage. Sci. Rep. 5, 8225 (2015)

15. DeBlase, C. R., Silberstein, K. E., Truong, T.-T., Abruña, H. D. \& Dichtel, W. R. $\beta$-Ketoenamine-linked covalent organic frameworks capable of pseudocapacitive energy storage. J. Am. Chem. Soc. 135, 16821-16824 (2013).

16. Ding, S.-Y. et al. Construction of covalent organic framework for catalysis: Pd/ COF-LZU1 in Suzuki-Miyaura coupling reaction. J. Am. Chem. Soc. 133, 19816-19822 (2011)

17. Xu, H. et al. Catalytic covalent organic frameworks via pore surface engineering. Chem. Commun. 50, 1292-1294 (2014)

18. Fang, Q. et al. 3D microporous base-functionalised covalent organic frameworks for size-selective catalysis. Angew. Chem. Int. Ed. 53, 2878-2882 (2014)

19. Shinde, D. B., Kandambeth, S., Pachfule, P., Kumar, R. R. \& Banerjee, R. Bifunctional covalent organic frameworks with two dimensional organocatalytic micropores. Chem. Commun. 51, 310-313 (2015).

20. Kuhn, P., Antonietti, M. \& Thomas, A. Porous, covalent triazine-based frameworks prepared by ionothermal synthesis. Angew. Chem. Int. Ed. 47, 3450-3453 (2008)

21. Wan, S. et al. Covalent organic frameworks with high charge carrier mobility. Chem. Mater. 23, 4094-4097 (2011).

22. Ding, X. et al. An $n$-channel two-dimensional covalent organic framework. I. Am. Chem. Soc. 133, 14510-14513 (2011).

23. Spitler, E. L. \& Dichtel, W. R. Lewis acid-catalysed formation of twodimensional phthalocyanine covalent organic frameworks. Nat. Chem. 2 672-677 (2010)

24. Ding, X. et al. Synthesis of metallophthalocyanine covalent organic frameworks that exhibit high carrier mobility and photoconductivity. Angew. Chem. Int. Ed. 50, 1289-1293 (2011).

25. Feng, X. et al. High-rate charge carrier transport in porphyrin covalent organic frameworks: switching from hole to electron to ambipolar conduction. Angew. Chem. Int. Ed. 51, 2618-2622 (2011).

26. Jin, S. et al. Two-dimensional tetrathiafulvalene covalent organic frameworks: towards latticed conductive organic salts. Chem. Eur. J. 20, 14608-14613 (2014).

27. Dalapati, S. et al. An azine-linked covalent organic framework. J. Am. Chem. Soc. 135, 17310-17313 (2013)

28. Lukose, B., Kuc, A. \& Heine, T. The structure of layered covalent-organic frameworks. Chem. Eur. J. 17, 2388-2392 (2011).

29. Spitler, E. L. et al. A 2D covalent organic framework with 4.7-nm pores and insight into its interlayer stacking. J. Am. Chem. Soc. 133, 19416-19421 (2011).
30. Lukose, B., Kuc, A., Frenzel, J. \& Heine, T. On the reticular construction concept of covalent organic frameworks. Beilstein J. Nanotechnol. 1, 60-70 (2010).

31. Chen, L. et al. Photoelectric covalent organic frameworks: converting open lattices into ordered donor-acceptor heterojunctions. J. Am. Chem. Soc. 136, 9806-9809 (2014).

32. Feng, X. et al. An ambipolar conducting covalent organic framework with self-sorted and periodic electron donor-acceptor ordering. Adv. Mater. 24, 3026-3031 (2012).

33. Jin, S. et al. Charge dynamics in a donor-acceptor covalent organic framework with periodically ordered bicontinuous heterojunctions. Angew. Chem. Int. Ed 52, 2017-2021 (2013).

34. Dogru, M., Sonnauer, A., Gavryushin, A., Knochel, P. \& Bein, T. A covalent organic framework with $4 \mathrm{~nm}$ open pores. Chem. Commun. 47, 1707-1709 (2011)

35. Li, Z. et al. A 2D azine-linked covalent organic framework for gas storage applications. Chem. Commun. 50, 13825-13828 (2014).

36. Zhang, W. et al. Supramolecular linear heterojunction composed of graphite-like semiconducting nanotubular segments. Science 334, 340-343 (2011).

37. Dössel, L. F. et al. Synthesis and controlled self-assembly of covalently linked hexa-peri-hexabenzocoronene/perylene diimide dyads as models to study fundamental energy and electron transfer processes. J. Am. Chem. Soc. 134, 5876-5886 (2012).

38. Müller, B. S. \& Müllen, K. Expanding benzene to giant graphenes: towards molecular devices. Philos. Trans. A Math. Phys. Eng. Sci. 365, 1453-1472 (2007).

39. Accelrys, Material studio release notes, Release 4.4, Accelrys Software, San Diego (2008).

40. Aradi, B., Hourahine, B. \& Frauenheim, T. DFTB +, a sparse matrix-based implementation of the DFTB method. J. Phys. Chem. A 111, 5678-5684 (2007)

41. Düren, T., Millange, F., Férey, G., Walton, K. S. \& Snurr, R. Q. Calculating geometric surface areas as a characterization tool for metal-organic frameworks. J. Phys. Chem. C 111, 15350-15356 (2007).

42. Saeki, A., Koizumi, Y., Aida, T. \& Seki, S. Comprehensive approach to intrinsic charge carrier mobility in conjugated organic molecules, macromolecules, and supramolecular architectures. Acc. Chem. Res. 45, 1193-1202 (2012).

43. Seki, S., Saeki, A., Sakurai, T. \& Sakamaki, D. Charge carrier mobility in organic molecular materials probed by electromagnetic waves. Phys. Chem. Chem. Phys. 16, 11093-11113 (2014).

44. Yasutani, Y., Saeki, A., Fukumatsu, T., Koizumi, Y. \& Seki, S. Unprecedented high local charge-carrier mobility in P3HT revealed by direct and alternating current methods. Chem. Lett. 42, 19-21 (2013).

\section{Acknowledgements}

This work was supported by a Grant-in-Aid for Scientific Research (A) (24245030) from the Ministry of Education, Culture, Sports, Science and Technology, Japan (MEXT). S.D acknowledges the IMS research fellowship and is currently an International Research Fellow of the Japan Society for the Promotion of Science (JSPS).

\section{Author contributions}

D.J. supervised and supported the project. S.D. conducted the experiments. T.S. and S.S are responsible for the photoconductivity experiments. M.A. and S.I. are responsible for the DFTB calculations. S.J. and J.G. performed the structure simulations. H.X. performed theoretical BET surface-area simulations. S.D. and D.J. wrote the manuscript and discussed the results with the contributing authors.

\section{Additional information}

Supplementary Information accompanies this paper at http://nature.com/ naturecommunications

Competing financial interests: The authors declare no competing financial interests

Reprints and permission information is available online at http://npg.nature.com/ repreintsandpermission/

How to cite this article: Dalapati, S. et al. Rational design of crystalline supermicroporous covalent organic frameworks with triangular topologies. Nat. Commun. 6:7786 doi: 10.1038/ncomms8786 (2015).

This work is licensed under a Creative Commons Attribution 4.0 International License. The images or other third party material in this article are included in the article's Creative Commons license, unless indicated otherwise in the credit line; if the material is not included under the Creative Commons license users will need to obtain permission from the license holder to reproduce the material. To view a copy of this license, visit http://creativecommons.org/licenses/by/4.0/ 